ELECTRONIC RESEARCH ANNOUNCEMENTS OF THE AMERICAN MATHEMATICAL SOCIETY

Volume 8, Pages 35-46 (September 19, 2002)

S $1079-6762(02) 00103-8$

\title{
QUANTUM AFFINE ALGEBRAS, COMBINATORICS OF YOUNG WALLS, AND GLOBAL BASES
}

\author{
SEOK-JIN KANG AND JAE-HOON KWON
}

(Communicated by Efim Zelmanov)

\begin{abstract}
We construct the Fock space representation of quantum affine algebras using combinatorics of Young walls. We also show that the crystal basis of the Fock space representation can be realized as the abstract crystal consisting of proper Young walls. We then generalize Lascoux-Leclerc-Thibon algorithm to obtain an effective algorithm for constructing the global bases of basic representations.
\end{abstract}

\section{INTRODUCTION}

Let $U_{q}(\mathfrak{g})$ be a quantum affine algebra of type $A_{n}^{(1)}, A_{2 n-1}^{(2)}, D_{n}^{(1)}, A_{2 n}^{(2)}, D_{n+1}^{(2)}$ and $B_{n}^{(1)}$, and let $\Lambda$ be a dominant integral weight of level 1. In 44, Kang introduced a new family of combinatorial objects called the Young walls. The Young walls consist of colored blocks with various shapes built on a given ground state wall $Y_{\Lambda}$ and can be viewed as generalizations of (colored) Young diagrams.

The rules and patterns for building Young walls and the action of Kashiwara operators are given explicitly in terms of combinatorics of Young walls. Then the set $\mathcal{Y}(\Lambda)$ of proper Young walls becomes an abstract crystal for the quantum affine algebra $U_{q}(\mathfrak{g})$, and the subcrystal $\mathcal{Y}_{\circ}(\Lambda)$ consisting of reduced proper Young walls is isomorphic to the crystal $B(\Lambda)$ of the basic representation $V(\Lambda)$.

In this paper, we construct the Fock space representation $\mathcal{F}(\Lambda)$ of $U_{q}(\mathfrak{g})$ in a purely combinatorial way. More precisely, we take $\mathcal{F}(\Lambda)$ to be the $\mathbb{Q}(q)$-vector space spanned by the proper Young walls, and define the $U_{q}(\mathfrak{g})$-module action on $\mathcal{F}(\Lambda)$ using combinatorics of Young walls. Then $\mathcal{F}(\Lambda)$ becomes an integrable $U_{q}(\mathfrak{g})$-module in the category $\mathcal{O}^{\text {int }}$. The Fock space $\mathcal{F}(\Lambda)$ can be regarded as the $q$-deformed wedge space arising from a level 1 perfect representation [9].

We also show that the crystal of $\mathcal{F}(\Lambda)$ coincides with the abstract crystal $\mathcal{Y}(\Lambda)$ consisting of proper Young walls. Thus, we get an explicit decomposition of the Fock space $\mathcal{F}(\Lambda)$ into a direct sum of irreducible highest weight modules over $U_{q}(\mathfrak{g})$ by locating the maximal vectors in the $U_{q}(\mathfrak{g})$-crystal $\mathcal{Y}(\Lambda)$.

Finally, we generalize Lascoux-Leclerc-Thibon algorithm [10] to obtain an effective algorithm for constructing the global basis $G(\Lambda)$ of the basic representation

Received by the editors December 14, 2001.

2000 Mathematics Subject Classification. Primary 17B37; Secondary 17B10.

Key words and phrases. Quantized universal enveloping algebra, crystal basis, global basis.

This research was supported by KOSEF Grant \# 98-0701-01-05-L and the Young Scientist Award, Korean Academy of Science and Technology. 
$V(\Lambda)$. To each reduced proper Young wall $Y \in \mathcal{Y}_{\circ}(\Lambda)$, the corresponding global basis element $G(Y)$ can be expressed as a linear combination of proper Young walls whose coefficient polynomials form an upper triangular matrix with the diagonal entries 1 . We expect that there exist some interesting algebraic structures such that the irreducible modules at some specializations are parametrized by the reduced proper Young walls and that the decomposition matrices are determined by the polynomials giving the global basis elements.

\section{QuAntum AFFine ALGEBRAS}

Let $I=\{0,1, \ldots, n\}$ be an index set and let $\left(A, P^{\vee}, P, \Pi^{\vee}, \Pi\right)$ be an affine Cartan datum:

(i) $A=\left(a_{i j}\right)_{i, j \in I}$ is a generalized Cartan matrix of affine type,

(ii) $P^{\vee}=\mathbb{Z} h_{0} \oplus \cdots \oplus \mathbb{Z} h_{n} \oplus \mathbb{Z} d$ is the dual weight lattice,

(iii) $P=\left\{\lambda \in \mathfrak{h}^{*} \mid \lambda\left(P^{\vee}\right) \subset \mathbb{Z}\right\}$ is the weight lattice, where $\mathfrak{h}=\mathbb{Q} \otimes_{\mathbb{Z}} P^{\vee}$ is the Cartan subalgebra,

(iv) $\Pi^{\vee}=\left\{h_{i} \mid i \in I\right\}$ is the set of simple coroots,

(v) $\Pi=\left\{\alpha_{i} \mid i \in I\right\}$ is the set of simple roots defined by $\alpha_{j}\left(h_{i}\right)=a_{i j}, \alpha_{j}(d)=$ $\delta_{0, j}(i, j \in I)$.

Set $P^{+}=\left\{\lambda \in P \mid \lambda\left(h_{i}\right) \in \mathbb{Z}_{\geq 0}\right.$ for all $\left.i \in I\right\}$. The elements of $P^{+}$are called the dominant integral weights.

To each affine Cartan datum, we can associate an infinite-dimensional Lie algebra $\mathfrak{g}$ called the affine Kac-Moody algebra. The canonical central element and the null root of $\mathfrak{g}$ will be expressed as $c=\sum_{i=0}^{n} c_{i} h_{i}$ and $\delta=\sum_{i=0}^{n} d_{i} \alpha_{i}$, respectively, where $c_{i}$ and $d_{i}(i \in I)$ are positive integers given in [3. The fundamental weights $\Lambda_{i}$ $(i \in I)$ are the linear functionals defined by $\Lambda_{i}\left(h_{j}\right)=\delta_{i j}, \Lambda_{i}(d)=0(i, j \in I)$.

We denote by $q^{h}\left(h \in P^{\vee}\right)$ the basis elements of the group algebra $\mathbb{Q}(q)\left[P^{\vee}\right]$ with the multiplication $q^{h} q^{h^{\prime}}=q^{h+h^{\prime}}\left(h, h^{\prime} \in P^{\vee}\right)$. Let $(\mid)$ be a nondegenerate symmetric bilinear form on $\mathfrak{h}^{*}$ satisfying $\frac{2\left(\alpha_{i} \mid \alpha_{j}\right)}{\left(\alpha_{i} \mid \alpha_{i}\right)}=a_{i j}$ for $i, j \in I$. Set $q_{i}=q^{\frac{\left(\alpha_{i} \mid \alpha_{i}\right)}{2}}$, $K_{i}=q^{\frac{\left(\alpha_{i} \mid \alpha_{i}\right)}{2} h_{i}},[k]_{i}=\frac{q_{i}^{k}-q_{i}^{-k}}{q_{i}-q_{i}^{-1}}$ and $[n]_{i} !=\prod_{k=1}^{n}[k]_{i}$. We also use the notation $e_{i}^{(n)}=e_{i}^{n} /[n]_{i}$ ! and $f_{i}^{(n)}=f_{i}^{n} /[n]_{i} !$.

The quantum affine algebra $U_{q}(\mathfrak{g})$ is the associative algebra with 1 over $\mathbb{Q}(q)$ generated by $e_{i}, f_{i}(i \in I)$ and $q^{h}\left(h \in P^{\vee}\right)$ subject to the defining relations given, for example, in [6]. In this paper, we will focus on the quantum affine algebras of type $A_{n}^{(1)}, A_{2 n-1}^{(2)}, D_{n}^{(1)}, A_{2 n}^{(2)}, D_{n+1}^{(2)}$ and $B_{n}^{(1)}$.

\section{Crystal bases}

Recall that the category $\mathcal{O}^{\text {int }}$ consists of $U_{q}(\mathfrak{g})$-modules $M$ satisfying the properties:

(i) $M=\bigoplus_{\lambda \in P} M_{\lambda}$, where $M_{\lambda}=\left\{v \in M \mid q^{h} v=q^{\lambda(h)} v\right.$ for all $\left.h \in P^{\vee}\right\}$,

(ii) for each $i \in I, M$ is a direct sum of finite-dimensional irreducible $U_{(i)^{-}}$ modules, where $U_{(i)}=\left\langle e_{i}, f_{i}, K_{i}^{ \pm}\right\rangle \cong U_{q}\left(\mathfrak{s l}_{2}\right)$,

(iii) for any $v \in M$, there exists $l \geq 1$ such that $e_{i_{1}} \cdots e_{i_{l}} v=0$ for any $i_{1}, \ldots, i_{l} \in$ I.

For each $i \in I$, every element $u \in M_{\lambda}$ can be written uniquely as $u=\sum_{k \geq 0} f_{i}^{(k)} u_{k}$, where $k \geq-\lambda\left(h_{i}\right)$ and $u_{k} \in$ ker $e_{i} \cap M_{\lambda+k \alpha_{i}}$. The Kashiwara operators are the 
endomorphisms $\tilde{e}_{i}$ and $\tilde{f}_{i}$ on $M$ defined by

$$
\tilde{e}_{i} u=\sum_{k \geq 1} f_{i}^{(k-1)} u_{k}, \quad \tilde{f}_{i} u=\sum_{k \geq 0} f_{i}^{(k+1)} u_{k}
$$

Let $\mathbb{A}_{0}=\{f / g \in \mathbb{Q}(q) \mid f, g \in \mathbb{Q}[q], g(0) \neq 0\}$ be the localization of $\mathbb{Q}[q]$ at $q=0$.

Definition 3.1. A crystal basis of $M$ is a pair $(L, B)$, where

(i) $L$ is a free $\mathbb{A}_{0}$-module of $M$ such that $M \cong \mathbb{Q}(q) \otimes_{\mathbb{A}_{0}} L$,

(ii) $B$ is a $\mathbb{Q}$-basis of $L / q L$,

(iii) $L=\bigoplus_{\lambda \in P} L_{\lambda}$, where $L_{\lambda}=L \cap M_{\lambda}$,

(iv) $B=\bigsqcup_{\lambda \in P} B_{\lambda}$, where $B_{\lambda}=B \cap\left(L_{\lambda} / q L_{\lambda}\right)$,

(v) $\tilde{e}_{i} L \subset L, \tilde{f}_{i} L \subset L$ for all $i \in I$,

(vi) $\tilde{e}_{i} B \subset B \cup\{0\}, \tilde{f}_{i} B \subset B \cup\{0\}$ for all $i \in I$,

(vii) for $i \in I$ and $b, b^{\prime} \in B, \tilde{f}_{i} b=b^{\prime}$ if and only if $b=\tilde{e}_{i} b^{\prime}$.

The set $B$ becomes a colored oriented graph, called the crystal graph, with the arrows defined by $b \stackrel{i}{\rightarrow} b^{\prime}$ if and only if $\tilde{f}_{i} b=b^{\prime}$.

By extracting the properties of crystal graphs, we can define the notion of abstract crystals [7, 8]. An affine crystal is a set $B$ together with the maps wt : $B \rightarrow P$, $\varepsilon_{i}, \varphi_{i}: B \rightarrow \mathbb{Z} \cup\{-\infty\}, \tilde{e}_{i}, \tilde{f}_{i}: B \rightarrow B \cup\{0\}$ satisfying the conditions given in 7, 8].

Theorem $3.2([6])$.

(a) Let $V(\lambda)$ be the irreducible highest weight $U_{q}(\mathfrak{g})$-module with highest weight $\lambda \in P^{+}$and highest weight vector $u_{\lambda}$. Let $L(\lambda)$ be the free $\mathbb{A}_{0}$-submodule of $V(\lambda)$ spanned by the vectors of the form $\tilde{f}_{i_{1}} \cdots \tilde{f}_{i_{r}} u_{\lambda}\left(i_{k} \in I, r \in \mathbb{Z}_{\geq 0}\right)$ and set

$$
B(\lambda)=\left\{\tilde{f}_{i_{1}} \cdots \tilde{f}_{i_{r}} u_{\lambda}+q L(\lambda) \in L(\lambda) / q L(\lambda)\right\} \backslash\{0\} .
$$

Then $(L(\lambda), B(\lambda))$ is a crystal basis of $V(\lambda)$, and every crystal basis of $V(\lambda)$ is isomorphic to $(L(\lambda), B(\lambda))$.

(b) Define a $\mathbb{Q}$-algebra automorphism of $U_{q}(\mathfrak{g})$ by $\bar{e}_{i}=e_{i}, \bar{f}_{i}=f_{i}, \overline{q^{h}}=q^{-h}$ and $\bar{q}=q^{-1}$ for $i \in I$ and $h \in P^{\vee}$. Set $\mathbb{A}=\mathbb{Q}\left[q, q^{-1}\right]$ and let $V(\lambda)^{\mathbb{A}}=$ $U_{\mathbb{A}}^{-}(\mathfrak{g}) u_{\lambda}$, where $U_{\mathbb{A}}^{-}(\mathfrak{g})$ is the $\mathbb{A}$-subalgebra of $U_{q}(\mathfrak{g})$ generated by $f_{i}^{(n)}(i \in I$, $\left.n \in \mathbb{Z}_{\geq 0}\right)$. Then there exists a unique $\mathbb{A}$-basis $G(\lambda)=\{G(b) \mid b \in B(\lambda)\}$ of $V(\lambda)^{\mathbb{A}}$ such that

$$
G(b) \equiv b \quad \bmod q L(\lambda) \quad \text { and } \quad \overline{G(b)}=G(b)
$$

for all $b \in B(\lambda)$.

The basis $G(\lambda)$ is called the global basis or canonical basis of $V(\lambda)$ associated with the crystal graph $B(\lambda)$. 


\section{Combinatorics of Young Walls}

We briefly review the notion of Young walls and their combinatorics introduced by Kang [4]. The Young walls are built of colored blocks of three different shapes.

\begin{tabular}{c|c|c|c|c|c} 
Type & Shape & Width & Thickness & Height & Volume \\
\hline I & $\square=\square$ & 1 & 1 & 1 & 1 \\
II & $\square=\square$ & 1 & 1 & $\frac{1}{2}$ & $\frac{1}{2}$ \\
III & $\square=\square, \quad \square=\square$ & 1 & $\frac{1}{2}$ & 1 & $\frac{1}{2}$
\end{tabular}

Given a dominant integral weight $\Lambda$ of level 1, i.e. $\Lambda(c)=1$, we fix a frame $Y_{\Lambda}$ called the ground state wall of weight $\Lambda$ and on this frame, we build a wall of thickness less than or equal to one unit. The rules for building the walls are as follows:

(1) The colored blocks should be stacked in columns. No block can be placed on top of a column of half-unit thickness.

(2) Except for the right-most column, there should be no free space to the right of any block.

(3) The colored blocks should be stacked in a specified pattern which is determined by the type of the quantum affine algebra and $\Lambda$.

The coloring of blocks, description of ground state walls and the patterns for building the walls are given in [4]. For example, if $\mathfrak{g}=B_{3}^{(1)}$, and $\Lambda=\Lambda_{0}$, we will use the colored blocks

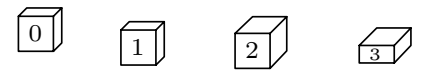

to build the walls on the ground state wall

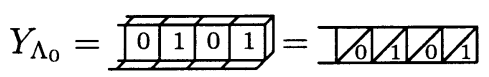

following the pattern:

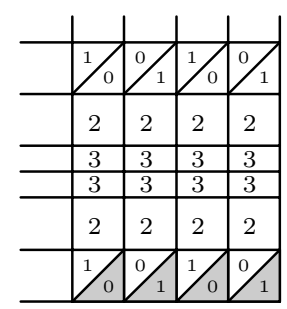

A wall built on $Y_{\Lambda}$ following the above rules is called a Young wall on $Y_{\Lambda}$, for the heights of its columns are weakly decreasing as we proceed from right to left. We often write $Y=\left(y_{k}\right)_{k=0}^{\infty}=\left(\ldots, y_{2}, y_{1}, y_{0}\right)$ as an infinite sequence of its columns.

\section{Definition 4.1.}

(1) A column of a Young wall is called a full column if its height is a multiple of the unit length and its top is of unit thickness.

(2) For quantum affine algebras of type $A_{2 n-1}^{(2)}, D_{n}^{(1)}, A_{2 n}^{(2)}, B_{n}^{(1)}$ and $D_{n+1}^{(2)}$, a Young wall is said to be proper if none of the full columns have the same heights. 
(3) For quantum affine algebras of type $A_{n}^{(1)}$, every Young wall is defined to be proper.

We will denote by $\mathcal{Y}(\Lambda)$ the set of all proper Young walls on $Y_{\Lambda}$.

Definition 4.2. Let $Y$ be a proper Young wall on $Y_{\Lambda}$.

(1) A block of color $i$ (in short, $i$-block) in $Y$ is called a removable $i$-block if $Y$ remains a proper Young wall after removing the block.

(2) A place in $Y$ is called an admissible $i$-slot if one may add an $i$-block to obtain another proper Young wall.

(3) A column in $Y$ is said to be $i$-removable (resp. $i$-admissible) if there is a removable $i$-block (resp. an admissible $i$-slot) in that column.

We now define the abstract Kashiwara operators $\tilde{E}_{i}, \tilde{F}_{i}$ on $\mathcal{Y}(\Lambda)$ as follows. Fix $i \in I$ and let $Y=\left(y_{k}\right)_{k=0}^{\infty}$ be a proper Young wall on $Y_{\Lambda}$.

(1) To each column $y_{k}$ of $Y$, we assign

$$
\begin{cases}-- & \text { if } y_{k} \text { is twice } i \text {-removable, } \\ - & \text { if } y_{k} \text { is once } i \text {-removable but not } i \text {-admissible } \\ -+ & \text { if } y_{k} \text { is once } i \text {-removable and once } i \text {-admissible, } \\ + & \text { if } y_{k} \text { is once } i \text {-admissible but not } i \text {-removable } \\ ++ & \text { if } y_{k} \text { is twice } i \text {-admissible, } \\ \cdot & \text { otherwise. }\end{cases}
$$

(2) From this sequence of +'s and -'s, we cancel out every $(+,-)$-pair to obtain a finite sequence of -'s followed by +'s, reading from left to right. This finite sequence $(-\cdots-,+\cdots+)$ is called the $i$-signature of $Y$.

(3) We define $\tilde{E}_{i} Y$ to be the proper Young wall obtained from $Y$ by removing the $i$-block corresponding to the right-most - in the $i$-signature of $Y$. We define $\tilde{E}_{i} Y=0$ if there is no - in the $i$-signature of $Y$.

(4) We define $\tilde{F}_{i} Y$ to be the proper Young wall obtained from $Y$ by adding an $i$-block to the column corresponding to the left-most + in the $i$-signature of $Y$. We define $\tilde{F}_{i} Y=0$ if there is no + in the $i$-signature of $Y$.

Next, we define

$$
\begin{aligned}
\operatorname{wt}(Y) & =\Lambda-\sum_{i \in I} k_{i} \alpha_{i} \in P, \\
\varepsilon_{i}(Y) & =\text { the number of }- \text { 's in the } i \text {-signature of } Y, \\
\varphi_{i}(Y) & =\text { the number of }+ \text { 's in the } i \text {-signature of } Y,
\end{aligned}
$$

where $k_{i}$ denotes the number of $i$-blocks in $Y$ that have been added to $Y_{\Lambda}$.

Proposition $4.3\left([4)\right.$. The set $\mathcal{Y}(\Lambda)$ together with the maps wt, $\varepsilon_{i}, \varphi_{i}, \tilde{E}_{i}$ and $\tilde{F}_{i}$ $(i \in I)$ becomes an affine crystal.

Let $\delta=d_{0} \alpha_{0}+\cdots+d_{n} \alpha_{n}$ be the null root of $U_{q}(\mathfrak{g})$, and set $a_{i}=d_{i}$ if $\mathfrak{g} \neq D_{n+1}^{(2)}$, $a_{i}=2 d_{i}$ if $\mathfrak{g}=D_{n+1}^{(2)}$. The part of a column with $a_{i}$-many $i$-blocks for each $i \in I$ in some cyclic order is called a $\delta$-column. A $\delta$-column in a proper Young wall is called removable if it can be removed to yield another proper Young wall.

Definition 4.4. A proper Young wall $Y$ is said to be reduced if none of its columns contain a removable $\delta$-column. 
Let $\mathcal{Y}_{\circ}(\Lambda) \subset \mathcal{Y}(\Lambda)$ be the set of all reduced proper Young walls on $Y_{\Lambda}$. Then we have:

Theorem $4.5\left([4)\right.$. The set $\mathcal{Y}_{\circ}(\Lambda)$ is an affine crystal. Moreover, there exists an affine crystal isomorphism $\mathcal{Y}_{\circ}(\Lambda) \stackrel{\sim}{\longrightarrow} B(\Lambda)$, where $B(\Lambda)$ is the crystal of the basic representation $V(\Lambda)$.

Remark 4.6. If $H_{N}(q)$ is a Hecke algebra of type $A_{N-1}$ with $q$ a primitive $n$th root of unity, then the irreducible representations of $H_{N}(q)$ are parametrized by the set of reduced proper Young walls of type $A_{n}^{(1)}$ with $N$ blocks [2]. We expect that there exist some interesting algebraic structures whose irreducible representations (at some specialization) are parametrized by reduced proper Young walls. In [1], Brundan and Kleshchev verified our speculation by showing that the irreducible representations of the Heck-Clifford superalgebra $H_{N}(q)$ with $q$ a primitive $(2 n+1)$ th root of unity are parametrized by the set of reduced proper Young walls of type $A_{2 n}^{(2)}$ with $N$ blocks.

\section{Fock SPACE REPRESENTATION}

Let $\mathcal{F}(\Lambda)=\bigoplus_{Y \in \mathcal{Y}(\Lambda)} \mathbb{Q}(q) Y$ be the $\mathbb{Q}(q)$-vector space with a basis $\mathcal{Y}(\Lambda)$. The goal of this section is to define a $U_{q}(\mathfrak{g})$-module structure on $\mathcal{F}(\Lambda)$, the Fock space representation of $U_{q}(\mathfrak{g})$. Moreover, we also show that the affine crystal $\mathcal{Y}(\Lambda)$ is exactly the crystal of $\mathcal{F}(\Lambda)$.

For this purpose, we need to define the action of $e_{i}, f_{i}(i \in I)$ and $q^{h}\left(h \in P^{\vee}\right)$ on proper Young walls in $\mathcal{Y}(\Lambda)$. Let $Y=\left(y_{k}\right)_{k=0}^{\infty}$ be a proper Young wall on $Y_{\Lambda}$. We denote by $\left|y_{k}\right|$ the number of blocks in $y_{k}$ added to $Y_{\Lambda}$. Then the associated partition is defined to be $|Y|=\left(\ldots,\left|y_{k}\right|, \ldots,\left|y_{1}\right|,\left|y_{0}\right|\right)$. For $Y=\left(y_{k}\right)_{k=0}^{\infty}, Z=\left(z_{k}\right)_{k=0}^{\infty}$ in $\mathcal{Y}(\Lambda)$, we define $|Y| \unrhd|Z|$ if and only if $\sum_{k=l}^{\infty}\left|y_{k}\right| \geq \sum_{k=l}^{\infty}\left|z_{k}\right|$ for all $l \geq 0$.

The action of $q^{h}\left(h \in P^{\vee}\right)$ on $Y \in \mathcal{Y}(\Lambda)$ is easily defined by $q^{h} Y=q^{\operatorname{wt}(Y)(h)}$. We now focus on the action of $e_{i}$ and $f_{i}$ on $Y(i \in I)$.

Case 1. Suppose that the $i$-blocks are of type I.

If $b$ is a removable $i$-block in $y_{k}$ of $Y$, then let $Y_{R}(b)=\left(y_{k-1}, \ldots, y_{1}, y_{0}\right)$ be the wall consisting of the columns lying on the right of $b$, and set $R_{i}(b ; Y)=$ $\varphi_{i}\left(Y_{R}(b)\right)-\varepsilon_{i}\left(Y_{R}(b)\right)$. (The wall $Y_{R}(b)$ should be regarded as a $U_{(i)}$-crystal, where no block can be added on $y_{l}$ for $l \geq k$.) We denote by $Y \nearrow b$ the Young wall obtained by removing $b$ from $Y$. Then we define

$$
e_{i} Y=\sum_{b} q_{i}^{-R_{i}(b ; Y)}(Y \nearrow b),
$$

where $b$ runs over all removable $i$-blocks in $Y$.

On the other hand, if $b$ is an admissible $i$-slot in $y_{k}$ of $Y$, then let $Y_{L}(b)=$ $\left(\ldots, y_{k+2}, y_{k+1}\right)$ be the Young wall consisting of the columns in $Y$ lying on the left of $b$, and set $L_{i}(b ; Y)=\varphi_{i}\left(Y_{L}(b)\right)-\varepsilon_{i}\left(Y_{L}(b)\right)$. (The wall $Y_{L}(b)$ may be a proper Young wall on another ground state wall $Y_{\Lambda^{\prime}}$.) We denote by $Y \measuredangle b$ the Young wall obtained by adding an $i$-block at $b$. Then we define

$$
f_{i} Y=\sum_{b} q_{i}^{L_{i}(b ; Y)}(Y \measuredangle b),
$$

where $b$ runs over all admissible $i$-slots in $Y$. 
Case 2. Suppose that the $i$-blocks are of type II (in this case, $q=q_{i}$ ).

Let $b$ be a removable $i$-block in $y_{k}$ of $Y$. If the $i$-signature of $y_{k}$ is -- , or if the $i$-signature of $y_{k}$ is - and there is another $i$-block below $b$, define $Y \nearrow b$ to be the Young wall obtained by removing $b$ from $Y$. If the $i$-signature of $y_{k}$ is -+ , or if the $i$-signature of $y_{k}$ is - and there is no $i$-block below $b$, define $Y \nearrow b=q^{-1}\left(1-\left(-q^{2}\right)^{l(b)+1}\right) Z$, where $Z$ is the Young wall obtained by removing $b$ from $Y$ and $l(b)$ is the number of $y_{l}$ 's with $l<k$ such that $\left|y_{l}\right|=\left|y_{k}\right|$.

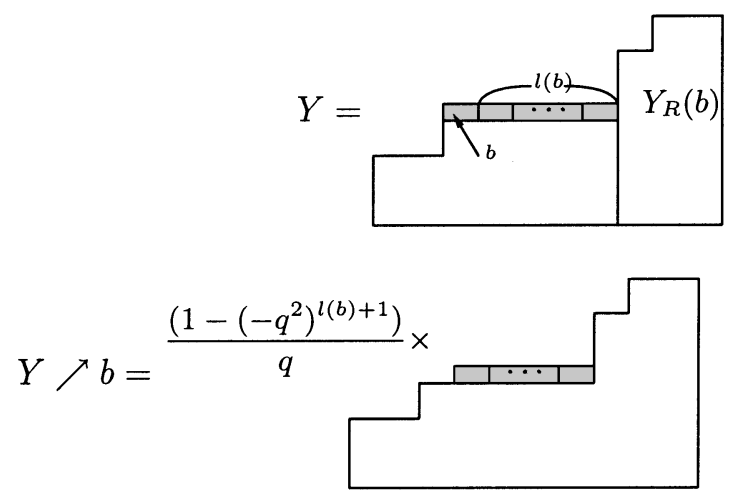

In either case, set $Y_{R}(b)=\left(y_{l-1}, \ldots, y_{0}\right)$, where $l$ is the integer such that $\left|y_{k}\right|=$ $\left|y_{k-1}\right|=\cdots=\left|y_{l+1}\right|<\left|y_{l}\right|$, and let $R_{i}(b ; Y)=\varphi_{i}\left(Y_{R}(b)\right)-\varepsilon_{i}\left(Y_{R}(b)\right)$. Then we define

$$
e_{i} Y=\sum_{b} q_{i}^{-R_{i}(b ; Y)}(Y \nearrow b)
$$

where $b$ runs over all removable $i$-blocks in $Y$.

On the other hand, suppose that $b$ is an admissible $i$-slot in $y_{k}$ of $Y$. If the $i$-signature of $y_{k}$ is ++ , or if the $i$-signature of $y_{k}$ is + and there is no $i$-block below $b$, then we define $Y \measuredangle b$ to be the Young wall obtained by adding an $i$-block at $b$. If the $i$-signature of $y_{k}$ is -+ , or if the $i$-signature of $y_{k}$ is + and there is another $i$-block below $b$, then we define $Y \measuredangle b=q^{-1}\left(1-\left(-q^{2}\right)^{l(b)+1}\right) Z$, where $Z$ is the Young wall obtained by adding an $i$-block at $b$ and $l(b)$ is the number of $y_{l}$ 's with $l>k$ such that $\left|y_{l}\right|=\left|y_{k}\right|$. That is

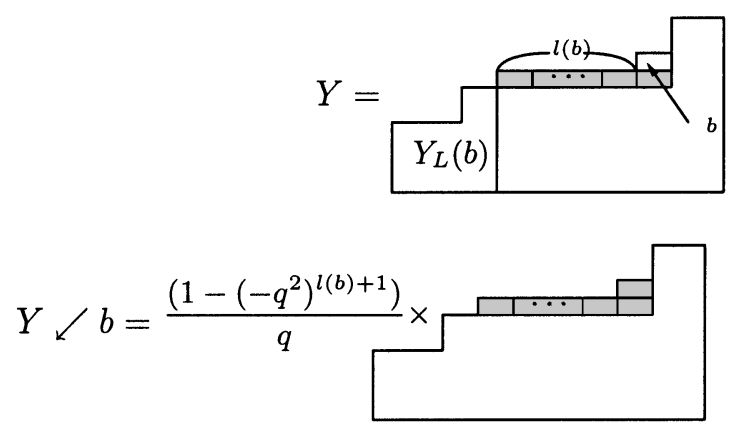

In either case, set $Y_{L}(b)=\left(\ldots, y_{l+2}, y_{l+1}\right)$, where $l$ is the integer such that $\left|y_{l+1}\right|<\left|y_{l}\right|=\left|y_{l-1}\right|=\cdots=\left|y_{k}\right|$, and let $L_{i}(b ; Y)=\varphi_{i}\left(Y_{L}(b)\right)-\varepsilon_{i}\left(Y_{L}(b)\right)$. Then 
we define

$$
f_{i} Y=\sum_{b} q_{i}^{L_{i}(b ; Y)}(Y \swarrow b),
$$

where $b$ runs over all admissible $i$-slots in $Y$.

Case 3. Suppose that the $i$-blocks are of type III.

If $b$ is a removable $i$-block in $y_{k}$ of $Y$, then we define $Y \nearrow b$ to be the Young wall obtained by removing $b$ from $Y$. We also consider the following $i$-block $b$ in $y_{k}$ of $Y$, which we call a virtually removable $i$-block.

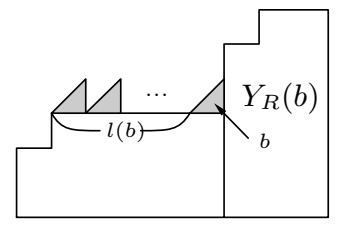

or

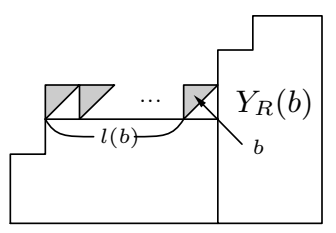

In this case, we define $Y \nearrow b$ to be
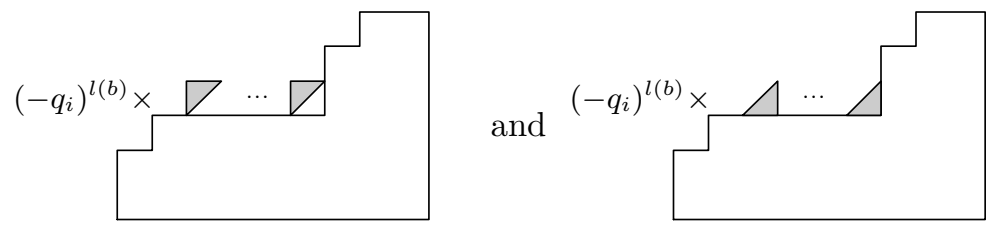

respectively, where $l(b)$ is given in the above figure. In either case, set $Y_{R}(b)=$ $\left(y_{k-1}, \ldots, y_{0}\right)$ and $R_{i}(b ; Y)=\varphi_{i}\left(Y_{R}(b)\right)-\varepsilon_{i}\left(Y_{R}(b)\right)$. Then we define

$$
e_{i} Y=\sum_{b} q_{i}^{-R_{i}(b ; Y)}(Y \nearrow b),
$$

where $b$ runs over all removable and virtually removable $i$-blocks in $Y$.

On the other hand, if $b$ is an admissible $i$-slot in $y_{k}$ of $Y$, then we define $Y \measuredangle b$ to be the Young wall obtained by adding an $i$-block at $b$. We also consider the following $i$-slot $b$ in $y_{k}$ of $Y$, which we call a virtually admissible $i$-slot

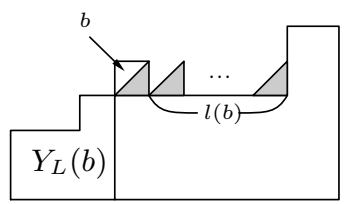

or

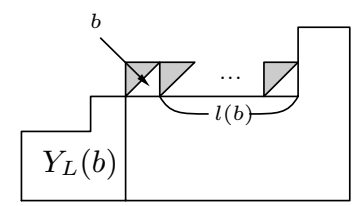

In this case, we define $Y \measuredangle b$ to be
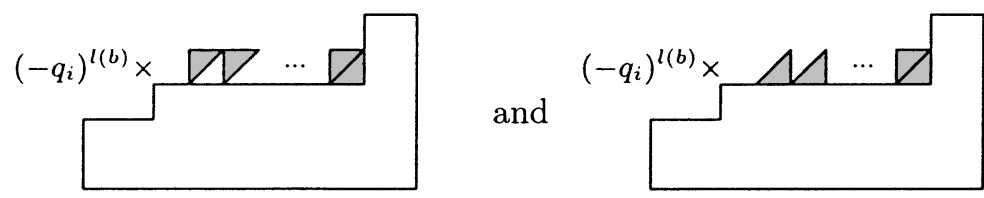

respectively, where $l(b)$ is given in the above figure. In either case, set $Y_{L}(b)=$ $\left(\ldots, y_{k+2}, y_{k+1}\right)$ and $L_{i}(b ; Y)=\varphi_{i}\left(Y_{L}(b)\right)-\varepsilon_{i}\left(Y_{L}(b)\right)$. Then we define

$$
f_{i} Y=\sum_{b} q_{i}^{L_{i}(b ; Y)}(Y \swarrow b),
$$


where $b$ runs over all admissible and virtually admissible $i$-slots in $Y$.

With these actions, we can verify that all the defining relations for the quantum affine algebra $U_{q}(\mathfrak{g})$ are satisfied. The verification is quite lengthy and based on the case-by-case check argument (see [5] for the details). Hence we obtain:

Theorem 5.1. The Fock space $\mathcal{F}(\Lambda)$ is an integrable $U_{q}(\mathfrak{g})$-module in the category $\mathcal{O}^{\text {int }}$.

It still remains to show that the affine crystal $\mathcal{Y}(\Lambda)$ is the crystal of $\mathcal{F}(\Lambda)$. Let $\mathcal{L}(\Lambda)=\bigoplus_{Y \in \mathcal{Y}(\Lambda)} \mathbb{A}_{0} Y$. It is easy to see that the pair $(\mathcal{L}(\Lambda), \mathcal{Y}(\Lambda))$ satisfies the first four conditions in Definition 3.1. For the remaining three conditions, the main step is to show that the Kashiwara operators on $\mathcal{Y}(\Lambda)$ induced by the $U_{q}(\mathfrak{g})$-module action on $\mathcal{F}(\Lambda)$ coincide with the abstract Kashiwara operators on $\mathcal{Y}(\Lambda)$ given in Section 4 . The proof of this step is based on the crystal basis theory for $U_{q}\left(\mathfrak{s l}_{2}\right)$ modules and the tensor product rule.

Theorem 5.2. The pair $(\mathcal{L}(\Lambda), \mathcal{Y}(\Lambda))$ is a crystal basis of the Fock space $\mathcal{F}(\Lambda)$, where $\mathcal{Y}(\Lambda)$ is the affine crystal given in Section 4.

Using Theorem 5.2, one can decompose the Fock space $\mathcal{F}(\Lambda)$ into a direct sum of irreducible highest weight modules over $U_{q}(\mathfrak{g})$ by locating the maximal vectors in the affine crystal $\mathcal{Y}(\Lambda)$.

\section{Corollary 5.3.}

$$
\mathcal{F}(\Lambda)= \begin{cases}\bigoplus_{m=0}^{\infty} V(\Lambda-m \delta)^{\oplus p(m)} & \text { if } \mathfrak{g} \neq D_{n+1}^{(2)}, \\ \bigoplus_{m=0}^{\infty} V(\Lambda-2 m \delta)^{\oplus p(m)} & \text { if } \mathfrak{g}=D_{n+1}^{(2)}\end{cases}
$$

\section{Generalized LLT algorithm}

In this section, we generalize Lascoux-Leclerc-Thibon algorithm to obtain an effective algorithm for constructing the global basis $G(\Lambda)$ of the basic representation $V(\Lambda)$. Note that $V(\Lambda)$ is realized as the $U_{q}(\mathfrak{g})$-submodule of $\mathcal{F}(\Lambda)$ generated by $Y_{\Lambda}$ and that the crystal $B(\Lambda)$ of $V(\Lambda)$ is isomorphic to the affine crystal $\mathcal{Y}_{\circ}(\Lambda)$ consisting of reduced proper Young walls. Thus our goal is the following: for each reduced proper Young wall $Y \in \mathcal{Y}_{\circ}(\Lambda)$, we would like to find an algorithm for the corresponding global basis element $G(Y)$ as a linear combination of proper Young walls in $\mathcal{Y}(\Lambda)$.

First, we describe the action of $f_{i}^{(r)}(i \in I)$ on $\mathcal{Y}(\Lambda)$. For a proper Young wall $Y \in \mathcal{Y}(\Lambda)$, write

$$
f_{i}^{(r)} Y=\sum_{\substack{Z \in \mathcal{Y}(\Lambda) \\ \operatorname{wt}(Z)=\operatorname{wt}(Y)-r \alpha_{i}}} Q_{Y, Z}(q) Z,
$$

where $Q_{Y, Z}(q) \in \mathbb{Q}(q)$. For each $Z=\left(z_{k}\right)_{k=0}^{\infty} \in \mathcal{Y}(\Lambda)$ with $Q_{Y, Z}(q) \neq 0$, there exists a unique sequence of Young walls $Y=Y^{(0)}, Y^{(1)}, \ldots, Y^{(r)}=Z$ such that

(i) $c_{k+1} Y_{k+1}=Y_{k} \swarrow b_{k+1}$ for a (virtually) admissible $i$-slot $b_{k+1}$ of $Y_{k}$ and $c_{k+1} \in \mathbb{Z}\left[q, q^{-1}\right]$

(ii) $b_{k+1}$ is placed on $b_{k}$ or to the right of $b_{k}$. 
We suppose that $b_{k}$ is located in the $i_{k}$ th column of $Y_{k-1}(1 \leq k \leq r)$. Let $Q_{Y^{(k)}, Y^{(k+1)}}(q)$ be the coefficient of $Y^{(k+1)}$ in the expression of $f_{i} Y^{(k)}$, and define

$$
Q_{Y, Z}^{\circ}(q)=\prod_{k=0}^{r-1} Q_{Y^{(k)}, Y^{(k+1)}}(q) \in \mathbb{Z}\left[q, q^{-1}\right] .
$$

If the $i$-blocks are of type II, consider

$$
\begin{aligned}
J_{1} & =\left\{k \mid b_{k-1} \text { is beneath } b_{k}\right\}, \\
J_{2} & =\left\{k \mid \text { there exists an } i \text {-block }\left(\neq b_{k-1}\right) \text { beneath } b_{k}\right\}, \\
J_{3} & =\left\{k \mid \text { there exists no } i \text {-block on and beneath } b_{k}\right\}, \\
S & =\left\{k \in J_{2} \mid k-1 \in J_{3} \text { and }\left|z_{i_{k-1}}\right|=\left|z_{i_{k}}\right|-1\right\} .
\end{aligned}
$$

Put $l=\left|J_{1}\right|, m=\left|J_{2}\right|$ and $n=\left|J_{3}\right|$. Note that $2 l+m+n=r$. For each $k \in S$, set $d_{k}=q c_{k}$.

Lemma 6.1. For $Y \in \mathcal{Y}(\Lambda)$, suppose that $Q_{Y, Z}(q) \neq 0$ and $\operatorname{wt}(Z)=\operatorname{wt}(Y)-r \alpha_{i}$ with $r \geq 1$ and $i \in I$. Let $\square$ be the type of the $i$-colored blocks. Then we have

$$
Q_{Y, Z}(q)= \begin{cases}Q_{Y, Z}^{\circ}(q) q_{i}^{\left(\begin{array}{c}
r \\
2
\end{array}\right)} & \text { if } \square=\mathrm{I}, \mathrm{III}, \\
Q_{Y, Z}^{\circ}(q) \frac{q^{\sigma(l, m, n)}}{[2]^{l} \prod_{k \in S} d_{k}} & \text { if } \square=\mathrm{II},\end{cases}
$$

where $\sigma(l, m, n)=4\left(\begin{array}{c}l \\ 2\end{array}\right)+\left(\begin{array}{c}m \\ 2\end{array}\right)+\left(\begin{array}{c}n \\ 2\end{array}\right)+2 l(m+n)+m n$. In particular, $Q_{Y, Z}(q) \in$ $\mathbb{Z}\left[q, q^{-1}\right]$.

Next, for a reduced proper Young wall $Y \in \mathcal{Y}_{\circ}(\Lambda)$, choose the left-most column $y_{l}$ such that $\left|y_{l}\right| \neq 0$ and denote by $b$ the top of $y_{l}$. Then, since $Y$ is reduced, $b$ must be a removable block of color, say, $i$. Remove $b$ from $Y$ and denote by $Y_{1}$ the resulting Young wall (if $y_{l}$ is twice $i$-removable, remove two $i$-blocks from $y_{l}$ ). If $Y_{1}$ is reduced, we stop there and set $\bar{Y}=Y_{1}$. If $Y_{1}$ is not reduced, then take the next $i$-removable block in $y_{l-1}$ and remove it from $Y_{1}$ to get $Y_{2}$ (if $y_{l-1}$ is twice $i$-removable, remove two $i$-blocks from $\left.y_{l-1}\right)$. We continue this process from left to right until we get a reduced proper Young wall and denote it by $\bar{Y}$. Suppose $\bar{Y}$ is obtained by removing $r$-many $i$-blocks from $Y$. If we write $f_{i}^{(r)} \bar{Y}=\sum_{Z} Q_{\bar{Y}, Z}(q) Z$, then by Lemma 6.1, it is easy to verify that $Q_{\bar{Y}, Y}(q)=1$.

By repeating the above process, we obtain a sequence of reduced proper Young walls $\left\{Y^{(k)}\right\}_{k=0}^{N}$, where $Y^{(0)}=Y, Y^{(1)}=\overline{Y^{(0)}}=\bar{Y}, \ldots, Y^{(k+1)}=\overline{Y^{(k)}}, \ldots$, $Y^{(N)}=\overline{Y^{(N-1)}}=Y_{\Lambda}$. Suppose that $Y^{(k+1)}=\overline{Y^{(k)}}$ is obtained by removing $r_{k+1^{-}}$ many $i_{k+1}$-blocks from $Y^{(k)}(0 \leq k \leq N-1)$. Then we define

$$
A(Y)=f_{i_{1}}^{\left(r_{1}\right)} \cdots f_{i_{N}}^{\left(r_{N}\right)} Y_{\Lambda} \in V(\Lambda)^{\mathbb{A}} .
$$

By definition, we have $\overline{A(Y)}=A(Y)$. Write $A(Y)=\sum_{Z \in \mathcal{Y}(\Lambda)} A_{Y, Z}(q) Z$, where $A_{Y, Z}(q) \in \mathbb{Q}(q)$. Then the coefficients $A_{Y, Z}(q)$ satisfy the following properties:

\section{Proposition 6.2.}

(a) $A_{Y, Z}(q) \in \mathbb{Z}\left[q, q^{-1}\right]$.

(b) $A_{Y, Z}(q)=0$ unless $|Y| \unrhd|Z|$.

(c) $A_{Y, Z}(q) \neq 0$ and $|Y|=|Z|$ imply $Y=Z$ and $A_{Y, Y}(q)=1$. 
Recall that there exists a lexicographic ordering $>$ on the set of partitions. For the proper Young walls with the same associated partition, we fix an arbitrary total ordering $\succ$. We now introduce a total ordering $>$ on the set $\mathcal{Y}(\Lambda)$ as follows: we define $Y>Z$ if either $|Y|>|Z|$ or $|Y|=|Z|$ and $Y \succ Z$. Note that if $|Y| \unrhd|Z|$ and $|Y| \neq|Z|$, then $Y>Z$.

By Proposition 6.2, $A(\Lambda)=\left\{A(Y) \mid Y \in \mathcal{Y}_{\circ}(\Lambda)\right\}$ are linearly independent over $\mathbb{Q}(q)$. Hence it is a $\mathbb{Q}(q)$-basis of $V(\Lambda)$. We claim that $A(\Lambda)$ is actually an $\mathbb{A}$-basis of $V(\Lambda)^{\mathbb{A}}$.

For a reduced proper Young wall $Y \in \mathcal{Y}_{\circ}(\Lambda)$, consider the corresponding global basis element

$$
G(Y)=\sum_{Z \in \mathcal{Y}(\Lambda)} G_{Y, Z}(q) Z
$$

Since $G(\Lambda)$ is an $\mathbb{A}$-basis of $V(\Lambda)^{\mathbb{A}}, G(Y)$ is an $\mathbb{A}$-linear combination of the vectors $f_{i_{1}}^{\left(r_{1}\right)} \cdots f_{i_{N}}^{\left(r_{N}\right)} Y_{\Lambda}$. Hence Lemma 6.1]implies $G_{Y, Z}(q) \in \mathbb{Q}\left[q, q^{-1}\right]$. Since $G(Y) \equiv Y$ $\bmod q \mathcal{L}(\Lambda)$, we may assume that $G_{Y, Z}(q) \in \mathbb{Q}[q]$ and $G_{Y, Z}(q) \in q \mathbb{Q}[q]$ unless $Y=Z$. Write $G(Y)=\sum_{W \in \mathcal{Y}_{\circ}(\Lambda)} H_{Y, W}(q) A(W)$, where $H=\left(H_{Y, W}(q)\right)_{Y, W \in \mathcal{Y}_{\circ}(\Lambda)}$ is the basis change matrix from $A(\Lambda)$ to $G(\Lambda)$ over $\mathbb{Q}(q)$.

Since $\overline{G(Y)}=G(Y)$ and $\overline{A(W)}=A(W)$ for all $Y, W \in \mathcal{Y}_{\circ}(\Lambda)$, we get $H_{Y, W}(q)=$ $H_{Y, W}\left(q^{-1}\right)$. Consider $G=\left(G_{Y, Z}(q)\right), H=\left(H_{Y, W}(q)\right)$ and $A=\left(A_{W, Z}(q)\right)$, which are square matrices indexed by $\mathcal{Y}_{\circ}(\Lambda)$ in decreasing total ordering. Since $G=H A$ and $A$ is upper unit-triangular, we have $H=G A^{-1}$, which implies $H_{Y, W}(q) \in \mathbb{A}$. Hence we obtain:

Proposition 6.3. $A(\Lambda)=\left\{A(Y) \mid Y \in \mathcal{Y}_{\circ}(\Lambda)\right\}$ is an $\mathbb{A}$-basis of $V(\Lambda)^{\mathbb{A}}$.

Recall that $G(Y)=\sum_{W \in \mathcal{Y}_{\circ}(\Lambda)} H_{Y, W}(q) A(W)$, and let $W$ be the Young wall which is maximal among the ones with $H_{Y, W}(q) \neq 0$ with respect to the total ordering $>$. By the maximality of $W$ and Proposition 6.2 (b), we have $G_{Y, W}(q)=$ $H_{Y, W}(q) A_{W, W}(q)=H_{Y, W}(q)$. Since $G_{Y, W}(q) \in \mathbb{Q}[q]$ and $G_{Y, W}(q)=H_{Y, W}(q)=$ $H_{Y, W}\left(q^{-1}\right)=G_{Y, W}\left(q^{-1}\right), G_{Y, W}(q)$ must be a constant. Moreover, since $G(Y) \equiv Y$ $\bmod q \mathcal{L}(\Lambda)$, we conclude that $Y=W$ and $G_{Y, Y}(q)=H_{Y, Y}(q)=1$. Therefore, by Proposition 6.2 (b), we can write

$$
G(Y)=\sum_{Y \geq W} H_{Y, W}(q) A(W)=Y+\sum_{Y>Z} G_{Y, Z}(q) Z .
$$

Now, we can apply the algorithm for computing $G(Y)$ as was done in [10. Fix a weight $\lambda \leq \Lambda$, and let $Y_{1}>\cdots>Y_{l}$ be all the reduced proper Young walls in $\mathcal{Y}_{\circ}(\Lambda)_{\lambda}$. Note that (14) implies $G\left(Y_{l}\right)=A\left(Y_{l}\right)$. Suppose that we have computed $G\left(Y_{k+1}\right), \ldots, G\left(Y_{l}\right)$. Also, from (14), $A\left(Y_{k}\right)$ is an A-linear combination of $G\left(Y_{k}\right), G\left(Y_{k+1}\right), \ldots, G\left(Y_{l}\right)$. For $k+1 \leq s \leq l$, if the coefficient of $Y_{s}$ in $A\left(Y_{k}\right)-\sum_{p=k+1}^{s-1} \gamma_{p}(q) G\left(Y_{p}\right)$ (or $A\left(Y_{k}\right)$ if $s=k+1$ ) is $\sum_{i=0}^{r} a_{i} q^{-i}+\sum_{j=1}^{r^{\prime}} b_{j} q^{j}$, then define $\gamma_{s}(q)=\sum_{i=1}^{r} a_{i}\left(q^{i}+q^{-i}\right)+a_{0}$. Then we obtain

$$
G\left(Y_{k}\right)=A\left(Y_{k}\right)-\gamma_{k+1}(q) G\left(Y_{k+1}\right)-\cdots-\gamma_{l}(q) G\left(Y_{l}\right) .
$$

To summarize, we have 
Theorem 6.4. For a reduced proper Young wall $Y \in \mathcal{Y}_{\circ}(\Lambda)$, the above algorithm yields the corresponding global basis element

$$
G(Y)=\sum_{Z \in \mathcal{Y}(\Lambda)} G_{Y, Z}(q) Z
$$

Moreover, the coefficients $G_{Y, Z}(q)$ satisfy the following conditions:

(i) $G_{Y, Z}(q) \in \mathbb{Z}[q]$,

(ii) $G_{Y, Z}(q)=0$ unless $|Y| \unrhd|Z|$,

(iii) $G_{Y, Y}(q)=1$ and $G_{Y, Z}(0)=0$ if $Y \neq Z$.

\section{REFERENCES}

[1] J. Brundan, A. Kleshchev, Hecke-Clifford superalgebras, crystals of type $A_{2 n}^{(2)}$ and modular branching rules for $\hat{S}_{n}$, Represent. Theory 5 (2001), 317-403 (electronic). MR 2002j:17024

[2] R. Dipper, G. James, Representations of Hecke algebras of general linear groups, Proc. London Math. Soc. 52 (1986), 20-52. MR 88b:20065

[3] V. G. Kac, Infinite-dimensional Lie algebras, Cambridge University Press, 3rd ed., Cambridge, 1990. MR 92k:17038

[4] S.-J. Kang, Crystal bases for quantum affine Lie algebras and combinatorics of Young walls, RIM-GARC preprint (2000) 00-2, Seoul National University, to appear in Proc. London Math. Soc.

[5] S.-J. Kang, J.-H. Kwon, Fock space representations of quantum affine algebras and generalized Lascoux-Leclerc-Thibon algorithm, math.QA/0208204.

[6] M. Kashiwara, On crystal bases of the q-analogue of universal enveloping algebras, Duke Math. J. 63 (1991), 465-516. MR 93b:17045

[7] M. Kashiwara, Crystal bases and Littelmann's refined Demazure character formula, Duke Math. J. 71 (1993), 839-858. MR 95b:17019

[8] M. Kashiwara, Crystal bases of modified quantized enveloping algebras, Duke Math. J. 73 (1994), 383-413. MR 95c:17024

[9] M. Kashiwara, T. Miwa, J.-U. H. Petersen, C. M. Yung, Perfect crystals and q-deformed Fock space, Selecta Math. 2 (1996), 415-499. MR 98f:17012

[10] A. Lascoux, B. Leclerc, J.-Y. Thibon, Hecke algebras at roots of unity and crystal bases of quantum affine algebras, Comm. Math. Phys. 181 (1996), 205-263. MR 97k:17019

[11] G. Lusztig, Canonical bases arising from quantized enveloping algebras, J. Amer. Math. Soc. 3 (1990), 447-498. MR 90m:17023

School of Mathematics, Korea Institute for Advanced Study, Seoul 130-012, Korea E-mail address: sjkang@kias.re.kr

School of Mathematics, Korea Institute for Advanced Study, Seoul 130-012, Korea

E-mail address: jhkwon@kias.re.kr 